\title{
Imaging photoelectron photoion coincidence spectroscopy with velocity focusing electron optics
}

\author{
Andras Bodii, ${ }^{1, a)}$ Melanie Johnson, ${ }^{1}$ Thomas Gerber, ${ }^{1}$ Zsolt Gengeliczki, ${ }^{2}$ Bálint Sztáray, ${ }^{3}$ \\ and Tomas Baer ${ }^{4}$ \\ ${ }^{1}$ Paul Scherrer Institut, Villigen 5232, Switzerland \\ ${ }^{2}$ Institute of Chemistry, Eötvös Loránd University, Budapest 1117, Hungary \\ ${ }^{3}$ Department of Chemistry, University of the Pacific, Stockton, California 95211, USA \\ ${ }^{4}$ Department of Chemistry, University of North Carolina, Chapel Hill, North Carolina 27599, USA
}

(Received 9 November 2008; accepted 23 January 2009; published online 3 March 2009)

\begin{abstract}
An imaging photoelectron photoion coincidence spectrometer at the vacuum ultraviolet (VUV) beamline of the Swiss Light Source is presented and a few initial measurements are reported. Monochromatic synchrotron VUV radiation ionizes the cooled or thermal gas-phase sample. Photoelectrons are velocity focused, with better than $1 \mathrm{meV}$ resolution for threshold electrons, and also act as start signal for the ion time-of-flight analysis. The ions are accelerated in a relatively low, $40-80 \mathrm{~V} \mathrm{~cm}^{-1}$ field, which enables the direct measurement of rate constants in the $10^{3}-10^{7} \mathrm{~s}^{-1}$ range. All electron and ion events are recorded in a triggerless multiple-start/multiple-stop setup, which makes it possible to carry out coincidence experiments at $>100 \mathrm{kHz}$ event frequencies. As examples, the threshold photoelectron spectrum of the argon dimer and the breakdown diagrams for hydrogen atom loss in room temperature methane and the chlorine atom loss in cold chlorobenzene are shown and discussed. ( 2009 American Institute of Physics. [DOI: 10.1063/1.3082016]
\end{abstract}

\section{INTRODUCTION}

Energy-selected ions have been prepared and analyzed with photoelectron photoion coincidence spectroscopy (PEPICO) for slightly more than 40 years. ${ }^{1-6}$ Linear momenta and energy are conserved in photoionization processes, thus, the internal energy of the parent ion is defined by the initial internal energy of the neutral species, the photon energy, the ionization energy, and the kinetic energy of the electron. Energy selected electrons are used as a start signal for measuring the ion time-of-flight (TOF) distribution. Two different approaches have been employed for electron energy analysis: (a) a fixed energy light source is used and the electron energies are selected by a dispersive analyzer $^{1,2}$ or (b) a tunable light source is employed and only threshold electrons are detected, a process often termed as threshold PEPICO or TPEPICO. ${ }^{3,7-11}$ Benefits of the TPEPICO technique are the much higher collection efficiency for threshold electrons than for kinetic ones (better than $30 \%$ ), as well as higher electron energy resolution (better than $1 \mathrm{meV}$ possible). The major disadvantage is the contamination of the threshold electron signal by energetic electrons (hot electrons), which are difficult to distinguish from true threshold electrons by simple line of sight analyzers that discriminate electrons on the basis of their angular divergence.

Several approaches have been used to eliminate the hot electron contamination. In the first one, an achromatic electron extraction lens is followed by a dispersive analyzer, which stops most, but not all hot electrons. ${ }^{12}$ More recently,

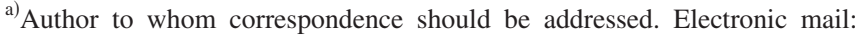
andras.boedi@psi.ch.
}

Sztáray and Baer ${ }^{5}$ developed a velocity focusing optics that focuses threshold electrons onto a small aperture and uses a second, off-axis aperture close by that collects only energetic electrons. By subtracting the hot electron PEPICO signal from the threshold signal, they obtained clean threshold coincidence spectra limited by the $10 \mathrm{meV}$ monochromator resolution. Although this scheme has been proven to work to within $10 \mathrm{meV}^{6}$ its applicability for photoelectron spectra rich in peaks is determined by the distance between the two apertures, which is ultimately limited by the Channeltron ${ }^{\circledR}$ diameter.

A pulsed photon source, such as a synchrotron storage ring operated, e.g., in single bunch mode, can also be used to select true threshold electrons by their TOF. ${ }^{13,14}$ However, the very low extraction fields required for good electron energy resolution and therefore the need to pulse ions out of the ionization region results in a structured false coincidence spectrum that is not easily separated from the true coincidences. A different approach, using pulsed field ionization (PFI) was pioneered by $\mathrm{Ng}$ and co-workers ${ }^{15-17}$ at the Advanced Light Source where they used the 112-140 ns dark gap in the multibunch mode to field ionize the high- $n$ Rydberg states and collected these by selectively gating the electronics to pass the PFI electrons while suppressing the prompt ones. Although this approach provides a very high resolution of $0.1 \mathrm{meV}$, it has the severe disadvantage that it also requires the use of very low electric fields that prohibit the ion from being efficiently extracted from the ionization region. Using the average extraction field of less than $5 \mathrm{~V} \mathrm{~cm}^{-1}$, the ion TOF distributions were not sharp and could not be used to analyze slow photodissociations. In ad- 
dition, the yield of PFI electrons was low. Nevertheless, this experiment set a standard for high resolution energy selection of ions.

In pulsed experiments, the need to minimize false coincidences constrains the experimental setup: the expected number of ionization events has to be around one per pulse, thereby lengthening the data acquisition time. In continuous single-start/single-stop (SS) and single-start/multiple-stop (SM) experiments, the count rates are still limited by the false coincidence background. It is only the multiple-start/ multiple-stop (MM) data acquisition technique that was shown to allow arbitrarily high ionization rates, ${ }^{18}$ as long as the detectors and the electronics are not paralyzed by the signal. In this report, we present a new imaging PEPICO experiment based on a quasicontinuous light source providing an energy resolution, $\Delta E / E=10^{-4}$, coupled to electron velocity focusing optics that is capable of sub-meV resolution for threshold electrons.

This imaging PEPICO (iPEPICO) at the X04DB vacuum ultraviolet (VUV) beamline of the Swiss Light Source (SLS) is designed to handle ionization event rates in excess of 100 $\mathrm{kHz}$ by recording all electron and ion signals. ${ }^{18}$ The electron velocity imaging detector enables electron kinetic energy analysis in the $0-400 \mathrm{meV}$ range with a $40 \mathrm{~V} \mathrm{~cm}^{-1}$ extraction field. The iPEPICO experiment is, thus, a cross between TPEPICO and PEPICO apparatuses with dispersive electron kinetic energy analyzers; the electron kinetic energy analysis and the ion TOF analysis are carried out simultaneously.

In the following sections, we describe the light source, the vacuum system, the electron and ion optics, and the data acquisition system, and illustrate the performance by reporting a few benchmark measurements. The main improvement with respect to previous synchrotron-based experiments is the use of higher extraction fields that provide TOF spectra with comparably good resolution and the simultaneous electron kinetic energy analysis with a better than $1 \mathrm{meV}$ resolution.

\section{EXPERIMENTAL}

\section{A. Light source}

Only a brief overview of the VUV beamline at the SLS is given here. The bending magnet radiation is collimated using a copper mirror with platinum reflective coating with a vertical acceptance of $4 \mathrm{mrad}$ and a horizontal acceptance of $8 \mathrm{mrad}$. Two silicon gratings, with 600 and $1200 \mathrm{~mm}^{-1}$ line density, are employed in the constant deviation configuration to produce monochromatic light with $10^{4}$ resolving power in the 5-15 and 15-30 eV energy ranges, respectively. The collimated beam is focused with a second mirror onto $110-1000 \mu \mathrm{m}$ vertical slits in the $80 \mathrm{~cm}$ long gas filter, $26 \mathrm{~m}$ from the source. The gas filter has eight differentially pumped chambers. The sixth chamber can be filled with 30 mbar rare gas in order to filter out higher harmonics above $15.759 \mathrm{eV}(10 \mathrm{mbar}, 75 \% \mathrm{Ne}, 25 \% \mathrm{Ar})$, or above $21.565 \mathrm{eV}$ (8 mbar Ne) without compromising the $10^{-10}-10^{-9} \mathrm{mbar}$ background pressure in the transfer line. For $h \nu<10 \mathrm{eV}$, a $\mathrm{MgF}_{2}$ window is used that absorbs virtually all light above
$11 \mathrm{eV}$. Above the Ne ionization energy, the beamline currently delivers light contaminated by higher harmonics up to $h \nu \approx 140 \mathrm{eV}$.

The exit slit position is determined by the focus position, and in order to obtain a high differential pumping efficiency, the rare gas pressure is largest where the apertures are the smallest, i.e., near the focus. This means the beam either has to be refocused, transferred by a glass capillary in the ionization region, or the ionization region has to be moved as close as possible to the focal point. At present, the latter two options are available and we typically operate with a relatively large spot size of $4 \times 2 \mathrm{~mm}^{2}$, which makes alignment easier than when using a capillary. Refocusing, at the expense of half the photon flux, would be beneficial if a smaller than $0.5 \times 0.5 \mathrm{~mm}^{2}$ spot size were desired.

The light intensity was measured to be approximately $10^{11} \mathrm{~s}^{-1}$, depending on the photon energy. This means that the light intensity, i.e., the ionization rate is several times that at the Lyman- $\alpha$ line at $1215.67 \AA$ with a conventional hydrogen discharge lamp, where coincidence data acquisition is typically impossible due to the high count rates. ${ }^{19}$ However, the high count rates are actually advantageous with the MM data acquisition technique.

\section{B. Continuous skimmed molecular beam source}

It is only possible to produce energy selected ions if the parent initial internal energy, the photon energy, and the ionization energy are known. If room temperature sample is used, the initial thermal energy contributes to the parent ion internal energy and may shift the photon energy at which the fragment ion appears, to lower energies, which leads to the thermal shift. Moderate to large size molecules not only have significant internal energy and hard to describe coordinates of internal motion (vibrations bordering on being hindered internal rotations, to name one), but also many degrees of freedom, which can potentially reduce the rate constants, thus lengthening the dissociation rates well beyond the TOF range of the parent. This results in the daughter ion appearing at higher energies than it would if the dissociation were fast. This effect is called the kinetic shift. Thermal and kinetic shifts typically arise in the same molecules, thereby complicating the accurate determination of dissociation energies.

The kinetic shift is inherent in statistical processes, but the thermal shift can be decreased by cooling the sample. Therefore, a well pumped source chamber was constructed to enable continuous molecular beams in the 1-3 normal $1 / \mathrm{h}$ gas flow range. As illustrated in Fig. 1, the sample enters the source chamber through a $10-100 \mu \mathrm{m}$ diameter aperture in a nozzle according to Buckland et al. ${ }^{20}$ The source chamber is pumped by a $50001 \mathrm{~s}^{-1}$ Leybold COOLVAC $5000 \mathrm{CL}$ cryopump and a $12501 \mathrm{~s}^{-1}$ Pfeiffer TPH 1201 UP turbomolecular pump. COOLVAC $5000 \mathrm{CL}$ has a 2000 normal liter capacity for Ar, which is equivalent to at least two weeks of uninterrupted operation. The sample enters the experimental chamber through the $1 \mathrm{~mm}$ opening of a Beam Dynamics skimmer, is ionized, and pumped by a $15001 \mathrm{~s} \mathrm{~s}^{-1}$ Leybold COOLVAC $1500 \mathrm{CL}$ cryopump and a $500 \mathrm{l} \mathrm{s}^{-1}$ Pfeiffer TMH 521 YP turbomolecular pump. The turbomolecular 


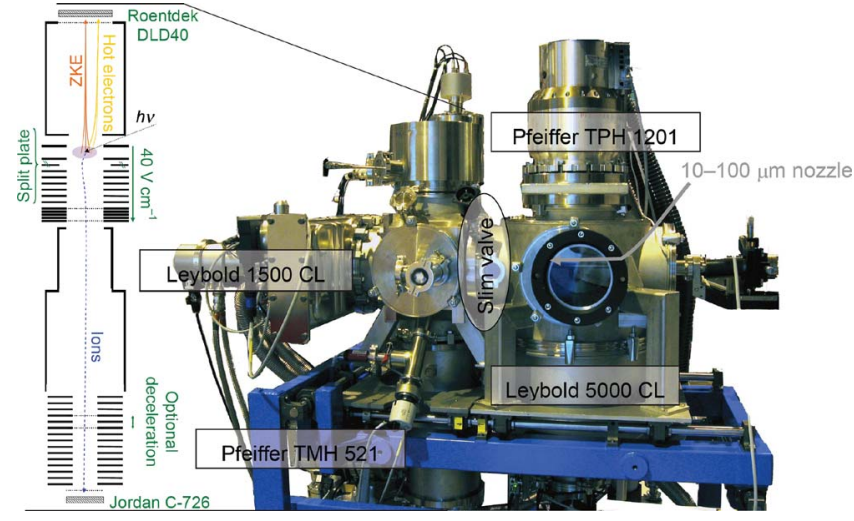

FIG. 1. (Color online) Overview of the iPEPICO experiment.

pumps are backed by an Adixen ACP 28 G series 2 Roots pump and the cryopumps are driven by a Leybold COOLPAK 6000D compressor unit and backed by an Adixen ACP $40 \mathrm{G}$ series 2 Roots pump. The total high vacuum pumping capacity of $82501 \mathrm{~s}^{-1}$ backed with an oil-free foreline system provides a clean vacuum system that minimizes the background when dealing with low density samples entrained in the molecular beam.

\section{Slim "clockwork" valve}

To maximize the molecular beam gas density at the ionization region, the skimmer has to be close to the ionization spot. This also means that the electrodes that extract the electrons and ions should have as small a diameter as possible consistent with minimizing field penetration. The other design criterion is that the source and the ionization chambers should be separated by a valve in order to enable (a) regeneration of the cryopump without flushing the source chamber, or (b) venting the ionization chamber without having to regenerate the COOLVAC 5000 CL cryopump. Commercial DN 200 valves are too thick and would place the skimmer too far from the ionization spot. The slim valves proposed by Chaban and Reutt-Robey, ${ }^{21}$ Stolow, ${ }^{22}$ and Küpper et $a l .{ }^{23}$ were all designed for high vacuum conditions and have only two states: open (through skimmer) and closed. Our vacuum criteria are looser, but we also wanted a third, open setting so that the molecular beam source can easily be converted into an effusive source. The clockwork valve, developed in the machine shop at the Paul Scherrer Institut and shown in Fig. 2 , has a dial with six possible positions. In its current configuration, two of the positions are used for skimmers, three are closed positions, and a fourth is fully open. Detailed dimensions for the valve are available. ${ }^{24}$ The skimmer is optically accessible directly through two DN 40 windows and obliquely through a DN 160 window on the source chamber. The valve protrudes slightly into the ionization chamber and the skimmer base to flange distance, reported by Küpper et al. to be $10 \mathrm{~mm}$ in their design, is only $3 \mathrm{~mm}$ in ours, albeit the skimmer base to valve end distance is $17 \mathrm{~mm}$. The seal is provided by an O-ring, against which the dial is pressed every time it is in position. O-ring tear so far appears to be less of an issue than had been feared. The skimmer has no mechanically fixed position and must be

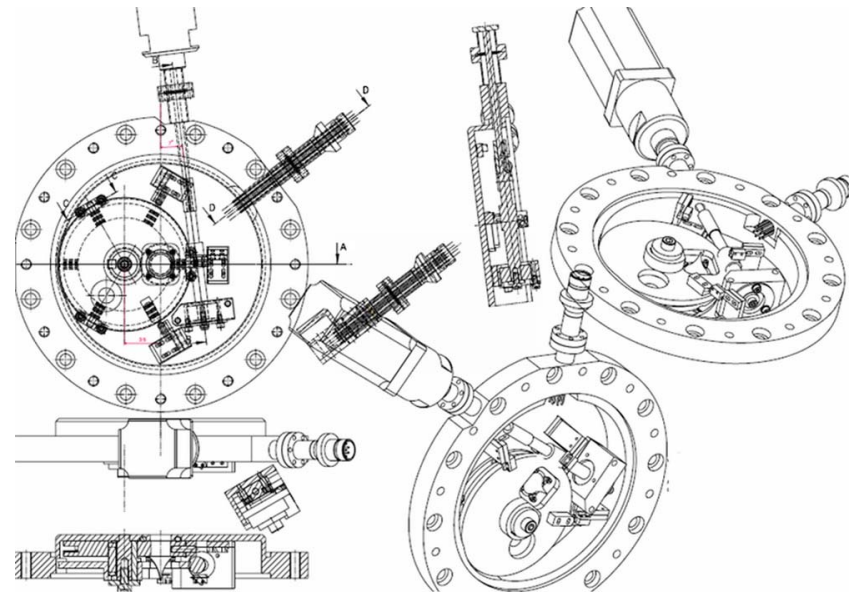

FIG. 2. (Color online) Schematic of the clockwork valve. For construction drawings see Ref. 24.

optimized together with the nozzle in a $1-2 \mathrm{~mm}$ range to maximize the molecular beam/light beam overlap.

\section{Electron and ion optics}

The electrons and ions are accelerated from ground in a $40-80 \mathrm{~V} \mathrm{~cm}^{-1}$ field, between two plates with $10 \mathrm{~mm}$ openings, separated by $11 \mathrm{~mm}$. The electrons fly vertically into the $265 \mathrm{~mm}$ long electron flight tube with a $20 \mathrm{~mm}$ opening, which serves to velocity image ${ }^{25-27}$ the beam at the $40 \mathrm{~mm}$ diameter Roentdek DLD40 delay line detector with resistance matched Photonics microchannel plates (MCPs) mounted in a Chevron configuration, $L: \operatorname{Det}=60: 1,25 \mu \mathrm{m}$ pore size, biased at $8^{\circ}$ from the front plate. Since a relatively low extraction field is needed to ensure high residence times of the ions in the acceleration region to measure dissociation rate constants, we opted to keep the velocity imaging optics simple, and have not included a magnifying lens to allow for higher fields. ${ }^{28}$ Electrons with zero transverse momentum are focused to a less than $1 \mathrm{~mm}$ diameter spot at the center of the detector.

The center spot of a zero kinetic energy electron image is shown in Fig. 3. The photon energy was decreased to approximately $4 \mathrm{meV}$ below the $\operatorname{Ar} I E$ to obtain a pure threshold electron signal, taking into account the photon energy resolution and the Stark shift. ${ }^{29}$ The spot full width at half maximum (FWHM) is $0.9 \times 0.4 \mathrm{~mm}^{2}$ and the radial distribution function drops to half its maximum at $R=0.4 \mathrm{~mm}$, which corresponds to $0.2 \mathrm{meV}$ electron kinetic energy. This spot size is significantly larger than the MCP channel-tochannel distance $(32 \mu \mathrm{m})$ or the theoretical limit of the spatial resolution of the delay line $(13 \mu \mathrm{m})$, which has been shown to be practical limits to the spatial resolution, ${ }^{30}$ thus, it is determined by the quality of the velocity imaging optics, just as was observed by Janssen and co-workers ${ }^{31}$ for ion imaging in their femtosecond coincidence apparatus.

The kinetic energy electron contamination of the threshold signal at the center of the image can be subtracted based on the average count rate in a surrounding ring area. This approach is guaranteed not to produce artifacts, such as the concentration of the noise along a central axis encountered in the inverse Abel transformation, ${ }^{32}$ as long as the photon en- 


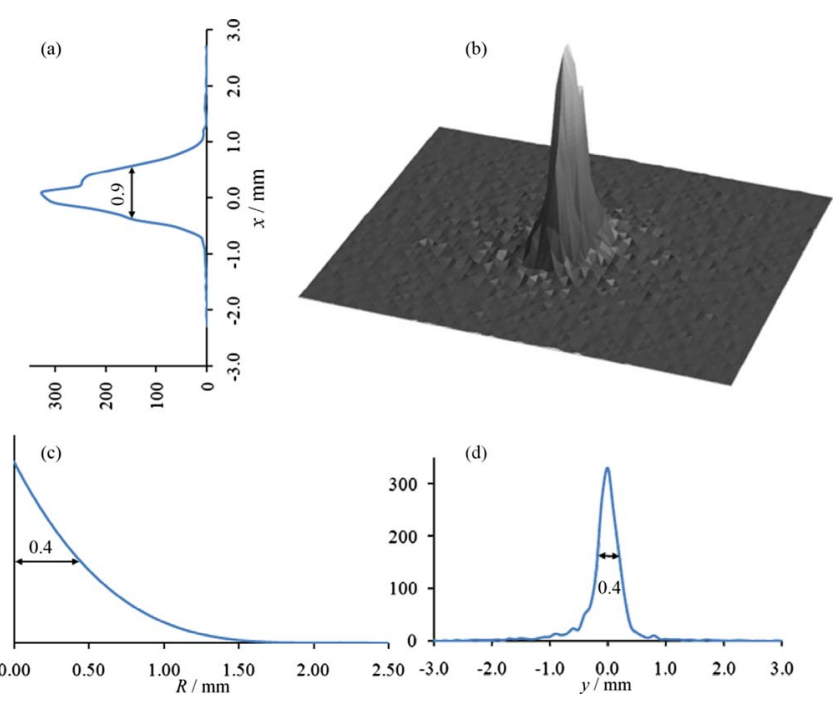

FIG. 3. (Color online) (b) Center threshold electron peak obtained with an extraction field of $80 \mathrm{~V} \mathrm{~cm}^{-1}$ of Ar with $x$ and $y$ projections [(a) and (d)] and (c) the radial distribution function. The $x$ and $y$ projections show counts accumulated over $120 \mathrm{~s}$ with $7 \times 10^{-7}$ mbar Ar pressure in the chamber and integrated over $0.3 \mathrm{~mm}$ along the $y$ and $x$ coordinates, respectively.

ergy resolution is commensurate with the expected electron kinetic energy in the ring area. There are two advantages to using the threshold electron signal. First, threshold electrons are focused onto the smallest area, which improves the $S / N$ ratio at energies where most electrons are highly energetic and the background in the electron image is high and almost constant. Second, electrons are focused onto a sphere, not uniformly in the detector plane, which means that focusing conditions will only be ideal for a given kinetic energy. Additionally, because of the $R \propto \sqrt{E}$ dependence of the ring radius on the kinetic energy, even constant focusing would entail a deteriorating absolute energy resolution at higher kinetic energies.

The kinetic energy range imaged at the detector depends on the focusing and the flight tube voltages. At a flight tube potential of $147 \mathrm{~V}$, electrons with approximately $400 \mathrm{meV}$ kinetic energy moving perpendicular to the flight axis are imaged at the rim of the active area of the detector at $15 \mathrm{~mm}$ radius (40 $\mathrm{V} \mathrm{cm}^{-1}$ extraction field). The energy range can be changed by scaling the extraction field and the corresponding flight tube potential. The electron image shown in Fig. 4 was obtained with the flight tube at $295 \mathrm{~V}\left(80 \mathrm{~V} \mathrm{~cm}^{-1}\right.$ extraction field), displays the $13 s^{\prime}$ autoionizing peak of Ar at $15.823 \mathrm{eV}$ (64 meV electrons), and also shows autoionization from $\mathrm{N}_{2}$ resulting in electrons with $178 \mathrm{meV}$ more kinetic energy. This is the two-dimensional projection of the threedimensional electron velocity distribution, i.e., the raw image, and only a higher limit to the FWHM of the two peaks is given based on their half width at half maximum on the high- $R$ side, which are 0.8 and $1.2 \mathrm{~mm}$, respectively, corresponding to at least 42 and $64 \mathrm{meV}$ electron energy resolutions at 4 and $8 \mathrm{~mm}$ off-center on the detector. The total active range of the detector corresponds to $870 \mathrm{meV}$ in this case. $\triangle E / e K E$ is estimated to be at most $33 \%$ and $26 \%$ for the two peaks, respectively, worse than the smallest $7 \%$ value reported by Neumark and co-workers. ${ }^{33}$ or the $3.5 \%$ by

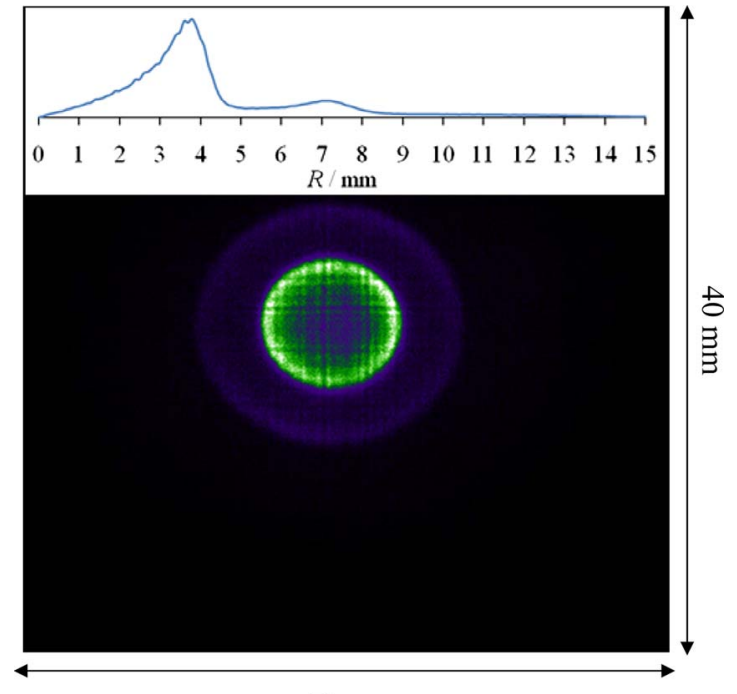

$40 \mathrm{~mm}$

FIG. 4. (Color online) False color electron image showing $\mathrm{Ar}$ and $\mathrm{N}_{2}$ autoionization features at $h \nu=15.822 \mathrm{eV}$. The inner ring corresponds to $64 \mathrm{meV}$ electrons emanating from $\mathrm{Ar}^{*}$ and the outer ring to $242 \mathrm{meV}$ electrons from $\mathrm{N}_{2}^{*}$. The radial distribution function is shown on top.

Janssen and co-workers. ${ }^{31}$ Our target function $\Delta E$ in the center over the energy range of the detector is, on the other hand, better than $1 / 2000$ (c.f. better than $1 / 200$ for the Neumark setup, see Fig. 3 in Ref. 33).

Two $\mu$-metal pieces, one encapsulating the whole electrode stack and the second one acting as the electron flight tube itself, provide magnetic shielding for the electrons. The magnetic shielding is incomplete at the electron detector side, but stray fields entering the electron flight tube through its opening do not seem to affect the image quality.

The second ion plate, located $12 \mathrm{~mm}$ from the ionization spot, has a $20 \mathrm{~mm}$ opening, and is cut in two "half-moon" sections so that a potential difference can be applied orthogonal to the ion extraction axis, parallel with the molecular beam axis. This transverse field enables us to correct the lateral offset of the ions at the detector caused by the initial transverse velocity in the molecular beam $\left(560 \mathrm{~m} \mathrm{~s}^{-1}\right.$ for the case of room temperature argon as a backing gas) and can increase the ion collection efficiency. However, this only becomes important at longer flight times in excess of $16 \mu \mathrm{s}$, i.e., a $9 \mathrm{~mm}$ transverse path at the $40 \mathrm{~mm}$ diameter Jordan MCP detector. After having flown $54 \mathrm{~mm}$ in the constant acceleration field, the ions are accelerated to $-550 \mathrm{~V}$ (in the case of $40 \mathrm{~V} \mathrm{~cm}^{-1}$ extraction field) within a space of $10 \mathrm{~mm}$. A ca. $550 \mathrm{~mm}$ long field-free section follows, at the end of which they are detected. The second half of the field-free drift region consists of 15 plates, to each of which a different voltage can be applied. It is, thus, possible, to decelerate ions and introduce a second field-free drift region at a lower potential, which can be used to distinguish daughter ions formed in slow dissociation reactions in the first field-free region. $^{34}$

\section{E. Data acquisition scheme}

Recording all detector events, i.e., all detected electrons and ions, not only shortens the acquisition time in continuous 
two-particle experiments, but also has two more advantages. It simplifies the false coincidence background subtraction, and makes it possible to measure at arbitrarily high count rates from the point of view of data analysis and signal to noise ratio. ${ }^{18}$ TOF acquisition schemes can be categorized as SS, SM, or MM. Conventional data acquisition techniques have been based on an SS (Ref. 6) or an SM (Refs. 13 and 15) scheme. However, in the high count rate environment of a synchrotron, it is essential to collect all starts and all stops in order to prevent paralysis of either the electron or the ion signal. We designed such a system based on a master clock that is used to time all detector events and correlate them in real time. The TDC8HP system by Roentdek, consisting of the HPTDC8 acquisition card by cronologic ${ }^{\mathrm{TM}} \mathrm{OHG}$ built on the CERN HPTDC chip, supports measurements of up to $2 \mathrm{~h}$ without trigger logic. To the best of our knowledge, this mode had not been realized before in coincidence experiments. ${ }^{35}$ The interface to the HPTDC 8 driver had to be rewritten in order to drive the acquisition card in the raw mode, in which all events are recorded and registered by software. Hits $\left(x_{1}, x_{2}, y_{1}, y_{2}\right.$, and MCP signals from the electron detector and ion hits from the ion detector) have timestamps, but are not necessarily read out in chronological order, therefore the events need to be ordered on the fly. The two $x$ and the two $y$ signals from the Roentdek detector are correlated in order to get the time stamp and the position information of the electron. Electron hits then need to be correlated with all ion signals (multiple stop) within the TOF range, irrespective of the readout sequence of the hits. Relevant sections of the Microsoft Visual $\mathrm{C}++$ code that interfaces between the HPTDC8 driver and the measurement user interface written in Delphi 2007 are available for download. ${ }^{24}$ The user interface was also integrated in the EPICS beamline control system, ${ }^{36}$ which enables continuous monitoring of the voltages, pressures, and fully automated data acquisition.

\section{RESULTS}

\section{A. Threshold photoelectron spectrum of $\mathrm{Ar}_{2}$}

The threshold photoelectron spectrum (TPES) of the argon dimer was recorded in the 14.5-15.8 eV energy range. $\mathrm{Ar}_{2}$ was prepared in a molecular beam using a $100 \mu \mathrm{m}$ nozzle at 1 bar Ar backing pressure. The pressures in the source and experimental chambers were $2.5 \times 10^{-4}$ and 1.6 $\times 10^{-6}$ mbar, respectively. The collection efficiencies for the electrons and ions were calculated at the Ar edge at 15.759 $\mathrm{eV}$ using $\eta_{e}=N_{c} / N_{i}=48 \%$ and $\eta_{i}=N_{c} / N_{e}=22 \%$, where $\eta_{e}$ and $\eta_{i}$ are the electron and ion collection efficiencies, and $N_{e}, N_{i}$, and $N_{c}$ are the measured electron, ion, and coincidence counts, respectively. The photon energies were calibrated with the autoionization peaks of $\operatorname{Ar}\left(11 s^{\prime}, 15.7639 \mathrm{eV}\right.$ and $\left.12 s^{\prime}, 15.7973 \mathrm{eV}\right)^{37}$ and $\mathrm{Ne}\left(13 s^{\prime}, 21.5619 \mathrm{eV}\right.$ and $12 d^{\prime}$ $21.5665 \mathrm{eV}),{ }^{38}$ by using the second harmonic radiation at half the $\mathrm{Ne}$ autoionization energies, and the $\mathrm{Xe} 8 s^{\prime}$ line $(12.5752 \mathrm{eV}) .{ }^{39}$ The photon energy resolution was measured to be $2 \mathrm{meV}$ using the autoionization peak widths.

$\mathrm{The} \mathrm{Ar}_{2}$ TPES and the photoionization efficiency curve for $\mathrm{Ar}_{2}^{+}$are shown in Fig. 5. The TPES can be collected with

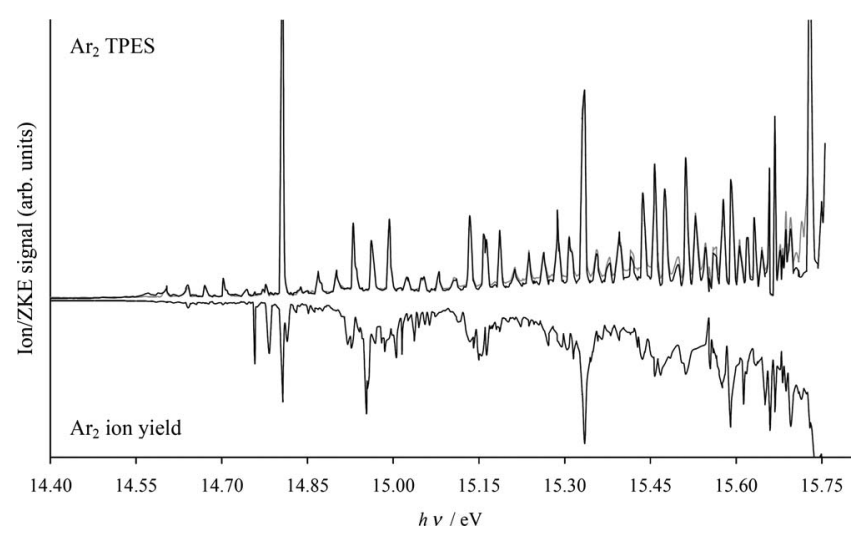

FIG. 5. TPES of $\mathrm{Ar}_{2}$. The dark line is the hot electron corrected zero kinetic energy electron (ZKE) signal, collected in coincidence with $\mathrm{Ar}_{2}^{+}$ions. The gray line gives the total hot electron corrected ZKE signal. The photon energy resolution is $2 \mathrm{meV}$. The lower curve is the total ion signal in which the peaks also correspond to autoionization processes.

or without coincidence with mass selected $\mathrm{Ar}_{2}^{+}$ions. There is little difference between the two spectra except for the slightly worse signal to noise for the mass selected one, which shows that e.g., Ar trimers are not formed and do not contribute to the TPES signal. However, for samples that consist of a mixture of various species that ionize in the same photon energy range, the TPES in coincidence with mass analyzed ions provides a route for collecting clean spectra. The peak positions and intensities agree well with the high resolution study of Signorell and Merkt. ${ }^{40}$ Although our resolution is far from theirs $\left(0.1-0.8 \mathrm{~cm}^{-1}\right.$, i.e., $\left.12-100 \mu \mathrm{eV}\right)$, the total measurement time with our setup amounted to less than a day, which is an appealingly short time for a spectral survey. Finally, as the photoelectron spectrum from 0 to 400 $\mathrm{meV}$ and the angular electron distributions are always recorded, it will also be possible to use these data to look at autoionizing states and determine their final ion state.

\section{B. $\mathrm{H}$-atom loss from $\mathrm{CH}_{4}^{+}$}

The iPEPICO setup was tested on the small methane molecule that dissociates rapidly on the time scale of the ion TOF. Both effusive and molecular beam sample sources were used. The room temperature breakdown diagram, which is a plot of the fractional abundance of the parent and fragment ion signals as a function of the photon energy, is available as electronic supplemental material. ${ }^{24}$ The slowly descending parent ion signal and the corresponding ascending methyl ion signal is a result of the thermal rotational distribution of the sample at $298 \mathrm{~K}$.

\section{Cl-atom loss from $\mathrm{C}_{6} \mathrm{H}_{5} \mathrm{Cl}^{+}$}

Stevens et al. ${ }^{41}$ measured and modeled the dissociation rates of halogen atom loss from halobenzenes over a wide internal energy range. We used $2 \% \mathrm{C}_{6} \mathrm{H}_{5} \mathrm{Cl}$ seeded in 1 bar Ar with a $100 \mu \mathrm{m}$ nozzle in the molecular beam source to reproduce their results and measure the temperature of the sample in the molecular beam. The optimized breakdown diagram, assuming a sample temperature of $100 \mathrm{~K}$ and yielding a best fit RRKM barrier of $3.235 \mathrm{eV}$, is shown in Fig. 6 . The breakdown diagram and the TOF distributions were fit 


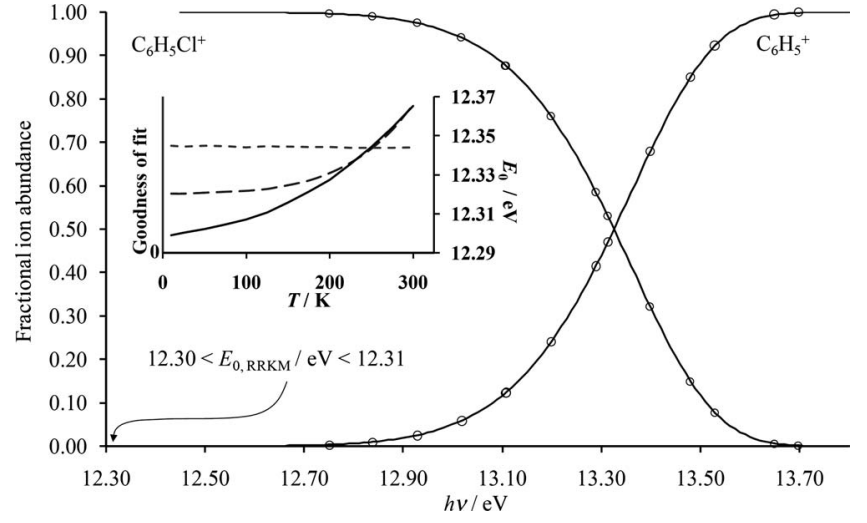

FIG. 6. Breakdown diagram of chlorobenzene in a molecular beam. The RRKM model of Stevens et al. (Ref. 41) and an ionization energy of 9.0728 $\mathrm{eV}$ (Ref. 43) was used in the model, and only the barrier was fit as a function of the assumed (equal vibrational and rotational) temperature in the molecular beam. The insert shows that the goodness of the TOF fit is independent of the assumed temperature, but both the goodness of the breakdown diagram and the barrier height change. Based on the goodness of the breakdown diagram fit, $125 \mathrm{~K}$ is an upper limit to the temperature, which leads to a $1 \mathrm{~kJ} \mathrm{~mol}^{-1}$ uncertainty in the barrier height.

using the reported $k(E)$ function, ${ }^{41}$ and only the internal temperature of chlorobenzene in the molecular beam and the barrier to dissociation were varied. As shown in the insert of Fig. 6, the statistical goodness of fit (short dashed lines) of the model to the TOF distributions, which are shown in the electronic supplemental material, ${ }^{24}$ did not vary with the assumed temperature. However, the goodness of the fit (long dashed lines) for the breakdown diagram began to increase (the fit became worse) above a temperature of $125 \mathrm{~K}$. This suggests that the sample temperature is below $125 \mathrm{~K}$, at which temperature the sample contains only $2.1 \mathrm{~kJ} / \mathrm{mol}$ $(22 \mathrm{meV})$ of rovibrational energy, compared to a room temperature average energy of $104 \mathrm{meV}$. The insensitivity of the breakdown diagram to temperature below $125 \mathrm{~K}$ is because the major broadening of the breakdown diagram is a result of the slow dissociation rates. In view of previously published studies on the cooling of vibrations in continuous molecular beams, ${ }^{42}$ a temperature around $100 \mathrm{~K}$ is not unreasonable, and the total $0-125 \mathrm{~K}$ temperature range only translates into $1 \mathrm{~kJ} \mathrm{~mol}^{-1}$ uncertainty in the barrier height. The measured and calculated TOF distributions (see Ref. 24) agree very well, which corroborates the reported $k(E)$ curve as well as our ability to measure slow dissociation rates.

\section{CONCLUSIONS}

The imaging PEPICO, originally proposed by Sztáray and Baer ${ }^{5}$ in 2003 has been designed and built at the Paul Scherrer Institut and is now operational at the VUV beamline of the Swiss Light Source. The main benefits of the experiment in comparison with the one described in Ref. 5 include a $1 \mathrm{meV}$ electron kinetic energy resolution in a continuous experiment, simultaneous electron kinetic energy analysis in the $0-800 \mathrm{meV}$ range, the use of tunable synchrotron radiation up to $30 \mathrm{eV}$ photon energy, and a large throughput pump system to allow for a continuous supersonic molecular beam. Successful applications of the iPEPICO setup described in this paper cover TPES (argon dimer), accurate appearance energies by means of TPEPICO ( $\mathrm{H}$ atom loss from methane), and measurement of slow rate constant in photoionization/ photodissociation processes of large molecules $(\mathrm{Cl}$ atom loss from chlorobenzene).

\section{ACKNOWLEDGMENTS}

We would like to thank Nicholas S. Shuman and Will Stevens for their assistance in assembling the experiment. The vacuum chambers and the optics were built in the workshop of the Paul Scherrer Institut and we owe special thanks to Steffen May for putting our ideas to paper. Members of the support team at the Swiss Light Source (especially Christian Lüscher, Peter Huber, and Gaiffi Nazareno) were always willing to lend us a hand. We thank Roentdek, Ottmar Jagutzki, and Achim Czasch for their help and guidance concerning the triggerless data acquisition scheme for the electron detector. The support of the Swiss Federal Office of Energy (BFE) is gratefully acknowledged (contract number 101969/152433). T.B. thanks the U.S. National Science Foundation (NSF Award Number CHE-0743000) and the U.S. Department of Energy (DOE Grant DE-FG0297ER14776). B.Sz. also wishes to thank the Hungarian Scientific Research Fund (OTKA Grant No. 71644) for support.

${ }^{1}$ B. Brehm and E. V. Puttkamer, Z. Naturforsch. 22A, 8 (1967).

${ }^{2}$ J. H. D. Eland, Int. J. Mass Spectrom. Ion Phys. 12, 389 (1973).

${ }^{3}$ R. Stockbauer, J. Chem. Phys. 58, 3800 (1973).

${ }^{4}$ A. S. Werner and T. Baer, J. Chem. Phys. 62, 2900 (1975).

${ }^{5}$ B. Sztáray and T. Baer, Rev. Sci. Instrum. 74, 3763 (2003).

${ }^{6}$ T. Baer, B. Sztáray, J. P. Kercher, A. F. Lago, A. Bodi, C. Skull, and D. Palathinkal, Phys. Chem. Chem. Phys. 7, 1507 (2005).

${ }^{7}$ K. Norwood and C. Y. Ng, J. Chem. Phys. 93, 6440 (1990).

${ }^{8}$ Q. Zha, R. N. Hayes, T. Nishimura, G. G. Meisels, and M. L. Gross, J. Phys. Chem. 94, 1286 (1990).

${ }^{9}$ R. Thissen, C. Alcaraz, J. W. Hepburn, M. Vervloet, and O. Dutuit, Int. J. Mass Spectrom. 199, 201 (2000).

${ }^{10}$ A. Bodi, J. P. Kercher, C. Bond, P. Meteesatien, B. Sztáray, and T. Baer, J. Phys. Chem. A 110, 13425 (2006).

${ }^{11}$ N. S. Shuman, M. A. Ochieng, B. Sztáray, and T. Baer, J. Phys. Chem. A 112, 5647 (2008).

${ }^{12}$ D. M. Smith, R. P. Tuckett, K. R. Yoxall, K. Codling, P. A. Hatherly, J. F. M. Aarts, and M. Stankiewicz, J. Chem. Phys. 101, 10559 (1994).

${ }^{13}$ O. Dutuit, T. Baer, C. Metayer, and J. Lemaire, Int. J. Mass Spectrom. Ion Process. 110, 67 (1991).

${ }^{14}$ J. W. Keister, T. Baer, R. Thissen, C. Alcaraz, O. Dutuit, H. Audier, and V. Troude, J. Phys. Chem. A 102, 1090 (1998).

${ }^{15}$ G. K. Jarvis, K. M. Weitzel, M. Malow, T. Baer, Y. Song, and C. Y. Ng, Rev. Sci. Instrum. 70, 3892 (1999).

${ }^{16}$ X. M. Qian, K. C. Lau, G. Z. He, C. Y. Ng, and M. Hochlaf, J. Chem. Phys. 120, 8476 (2004).

${ }^{17}$ G. K. Jarvis, R. C. Shiell, J. W. Hepburn, Y. Song, and C. Y. Ng, Rev. Sci. Instrum. 71, 1325 (2000).

${ }^{18}$ A. Bodi, B. Sztáray, T. Baer, M. Johnson, and T. Gerber, Rev. Sci. Instrum. 78, 084102 (2007).

${ }^{19}$ A. Bodi, B. Sztaray, and T. Baer, Phys. Chem. Chem. Phys. 8, 613 (2006).

${ }^{20}$ J. R. Buckland, R. L. Folkerts, R. B. Balsod, and W. Allison, Meas. Sci. Technol. 8, 933 (1997).

${ }^{21}$ E. E. Chaban and J. E. Reutt-Robey, Rev. Sci. Instrum. 64, 2391 (1993).

${ }^{22}$ A. Stolow, J. Vac. Sci. Technol. A 14, 2669 (1996).

${ }^{23}$ J. Küpper, H. Haak, K. Wohlfart, and G. Meijer, Rev. Sci. Instrum. 77, 016106 (2006)

${ }^{24}$ See EPAPS Document No. E-RSINAK-80-049902 for construction drawings for the slim valve, the breakdown diagram of $\mathrm{CH}_{4}$, and the experimental and fitted TOF distributions for $\mathrm{C}_{6} \mathrm{H}_{5} \mathrm{Cl}$ and $\mathrm{C}++$ code sections from the measurement software. For more information on EPAPS, see http://www.aip.org/pubservs/epaps.html.

${ }^{25}$ A. T. J. B. Eppink and D. H. Parker, Rev. Sci. Instrum. 68, 3477 (1997).

${ }^{26}$ D. W. Chandler and D. H. Parker, Adv. Photochem. 25, 59 (1999). 
${ }^{27}$ J. A. Davies, J. E. LeClaire, R. E. Continetti, and C. C. Hayden, J. Chem. Phys. 111, 1 (1999).

${ }^{28}$ H. F. Offerhaus, C. Nicole, F. Lepine, C. Bordas, F. Rosca-Pruna, and M. J. J. Vrakking, Rev. Sci. Instrum. 72, 3245 (2001).

${ }^{29}$ W. A. Chupka, J. Chem. Phys. 98, 4520 (1993).

${ }^{30}$ A. S. Tremsin, O. H. W. Siegmund, J. V. Vallerga, J. S. Hull, and R. Abiad, IEEE Trans. Nucl. Sci. 51, 1707 (2004)

${ }^{31}$ A. Vredenborg, W. G. Roeterdink, and M. H. M. Janssen, Rev. Sci. Instrum. 79, 063108 (2008).

${ }^{32}$ C. Bordas, F. Paulig, H. Helm, and D. L. Huestis, Rev. Sci. Instrum. 67, 2257 (1996)

${ }^{33}$ A. Osterwalder, M. J. Nee, J. Zhou, and D. M. Neumark, J. Chem. Phys. 121, 6317 (2004).

${ }^{34}$ J. Z. Davalos, H. Koizumi, and T. Baer, J. Phys. Chem. A 110, 5032 (2006).

${ }^{35}$ J. Ottmar and A. Czasch, personal communication (May 2008).
${ }^{36}$ See http://epics.web.psi.ch for more information about the implementation of EPICS at the Swiss Light Source.

${ }^{37}$ Y. Ralchenku, A. E. Kramida, J. Reader, and NIST ASD Team, NIST Atomic Spectra Database (National Institute of Standards and Technology, Gaithersburg, MD, 2008), http://physics.nist.gov/asd3.

${ }^{38}$ E. B. Saloman and C. J. Sansonetti, J. Phys. Chem. Ref. Data 33, 1113 (2004).

${ }^{39}$ E. B. Saloman, J. Phys. Chem. Ref. Data 33, 765 (2004).

${ }^{40}$ R. Signorell and F. Merkt, J. Chem. Phys. 109, 9762 (1998).

${ }^{41}$ W. Stevens, B. Sztáray, N. S. Shuman, T. Baer, and J. Troe, J. Phys. Chem. A 113, 573 (2009).

${ }^{42}$ P. M. Mayer and T. Baer, Int. J. Mass Spectrom. Ion Process. 156, 133 (1996).

${ }^{43}$ Y. Y. Youn, C. H. Kwon, J. C. Choe, and M. S. Kim, J. Chem. Phys. 117, 2538 (2002) 